Document downloaded from:

http://hdl.handle.net/10251/140253

This paper must be cited as:

Xu, S.; Chen, J.; Benítez López, J. (11-2). EP Elements in Rings with Involution. Bulletin of the Malaysian Mathematical Sciences Society. 42(6):3409-3426.

https://doi.org/10.1007/s40840-019-00731-x

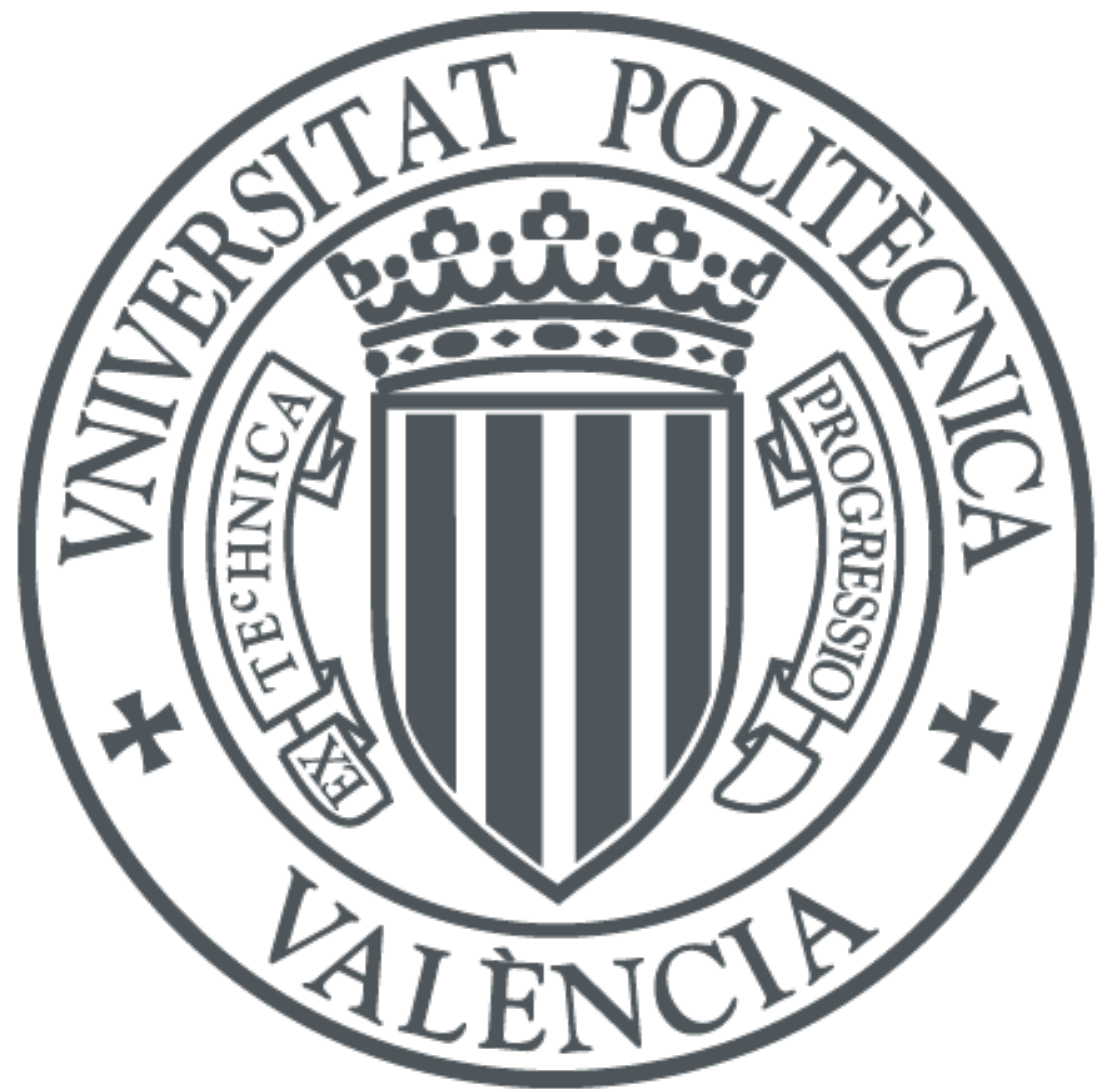

The final publication is available at

https://doi.org/10.1007/s40840-019-00731-x

Copyright Springer-Verlag

Additional Information 


\title{
EP elements in rings with involution
}

\author{
Sanzhang $\mathrm{Xu}$ and Jianlong Chen * \\ Department of Mathematics, Southeast University, Nanjing 210096, China
}

\section{Julio Benítez ${ }^{\dagger}$}

Universidad Politécnica de Valencia, Instituto de Matemática Multidisciplinar, Valencia, 46022, Spain

\begin{abstract}
Let $R$ be a unital ring with involution. We first show that the EP elements in $R$ can be characterized by three equations. Namely, let $a \in R$, then $a$ is EP if and only if there exists $x \in R$ such that $(x a)^{*}=x a, x a^{2}=a$ and $a x^{2}=x$. Any EP element in $R$ is core invertible and Moore-Penrose invertible. We give more equivalent conditions for a core (Moore-Penrose) invertible element to be an EP element. Finally, any EP element is characterized in terms of the $n$-EP property, which is a generalization of the bi-EP property.
\end{abstract}

Key words: Core inverse, EP, bi-EP, $n$-EP.

AMS subject classifications: 15A09, 16W10, $16 \mathrm{U} 80$.

\section{Introduction}

Throughout this paper, $R$ will denote a unital ring with involution, i.e., a ring with a mapping $a \mapsto a^{*}$ satisfying $\left(a^{*}\right)^{*}=a,(a b)^{*}=b^{*} a^{*}$ and $(a+b)^{*}=a^{*}+b^{*}$. The notion of core inverse for a complex matrix was introduced by Baksalary and Trenkler [3]. In [17], Rakić et al. generalized the core inverse of a complex matrix to the case of an element in $R$. More precisely, let $a, x \in R$, if

$$
a x a=a, x R=a R \quad \text { and } \quad R x=R a^{*},
$$

then $x$ is called a core inverse of $a$. If such an element $x$ exists, then it is unique and denoted by $a^{\oplus}$. The set of all core invertible elements in $R$ will be denoted by $R^{\oplus}$. Also, in [17] the authors defined a related pseudo-inverse in a ring with an involution. If $a \in R$, then $a_{\circledast} \in R$ is called a core dual inverse of $a$ if

$$
a a_{\oplus} a=a, a_{\oplus} R=a^{*} R \quad \text { and } \quad R a_{\oplus}=R a .
$$

Rakić et al. proved that if $a$ has a core dual inverse (we say that $a$ is core dual invertible), then is unique. We denote by $R_{\circledast}$ the subset of $R$ composed of core dual invertible

\footnotetext{
*E-mail: xusanzhang5222@126.com. Corresponding author, E-mail: jlchen@seu.edu.cn

${ }^{\dagger}$ E-mail: jbenitez@mat.upv.es
} 
elements. It is elemental to prove that $a \in R^{\oplus}$ if an only if $a^{*} \in R_{\oplus}$, and in this case, one has $\left(a^{\boxplus}\right)^{*}=\left(a^{*}\right)_{\circledast}$. This last observation permits to get results concerning dual core inverses from the corresponding results on dual inverses.

Let $a, x \in R$. If

$$
a x a=a, \quad x a x=x, \quad(a x)^{*}=a x \quad \text { and } \quad(x a)^{*}=x a,
$$

then $x$ is called a Moore-Penrose inverse of $a$. If such an element $x$ exists, then it is unique and denoted by $a^{\dagger}$. The set of all Moore-Penrose invertible elements will be denoted by $R^{\dagger}$.

Let $a \in R$. It can be easily proved that the set of elements $x \in R$ such that:

$$
a x a=a, \quad x a x=x \quad \text { and } \quad a x=x a
$$

is empty or a singleton. If the set is a singleton, its unique element is called the group inverse of $a$ and denoted by $a^{\#}$. The set of all group invertible elements will be denoted by $R^{\#}$. The subset of $R$ composed of invertible elements will be denote by $R^{-1}$.

A matrix $A \in \mathbb{C}_{n \times n}$ is called an EP (range-Hermitian) matrix if the range equality $\mathcal{R}(A)=\mathcal{R}\left(A^{*}\right)$, where $\mathbb{C}_{n \times n}$ denotes the set of all $n \times n$ matrices over the field of complex numbers and $\mathcal{R}(A)$ stands for the range (column space) of $A \in \mathbb{C}_{n \times n}$. This concept was first introduced by Schwerdtfeger in [18]. An element $a \in R$ is said to be an EP element if $a \in R^{\dagger} \cap R^{\#}$ and $a^{\dagger}=a^{\#}[8]$. The set of all EP elements will be denoted by $R^{\mathrm{EP}}$. Mosić et al. in [12, Theorem 2.1] gave several equivalent conditions such that an element in $R$ to be an EP element. Patrício and Puystjens in [16, Proposition 2] proved that for a Moore-Penrose invertible element $a \in R, a \in R^{\mathrm{EP}}$ if and only if $a R=a^{*} R$. As for a Moore-Penrose invertible element $a \in R, a \in R^{\mathrm{EP}}$ if and only if $a a^{\dagger}=a^{\dagger} a$, thus we deduce that $a a^{\dagger}=a^{\dagger} a$ if and only if $a R=a^{*} R$. In [17, Theorem 3.1], Rakić et al. investigated some equivalent conditions such that a (dual) core invertible element in $R$ to be an EP element. Also, they showed that $R^{\dagger} \cap$ core $R=R^{\dagger} \cap R^{\#}$. Motivated by $[6,12,16,17]$, in this paper, we will give new equivalent characterizations such that an element in $R$ to be an EP element.

We first show that the EP elements in $R$ can be characterized by three equations. That is, let $a \in R$, then $a \in R^{\mathrm{EP}}$ if and only if there exists $x \in R$ such that $(x a)^{*}=x a$, $x a^{2}=a$ and $a x^{2}=x$. In [17], Rakić et al. proved that $a \in R^{\dagger}$ if and only if there exists $x \in R$ such that $a x a=a, x R=a^{*} R$ and $R x=R a^{*}$. Inspired by this result, we show that $a \in R^{\mathrm{EP}}$ if and only if there exists $x \in R$ such that

$$
a x a=a, x R=a R \quad \text { and } \quad R x^{*}=R a .
$$

In [6, Theorem 16], for an operator $T \in L(X)$, where $X$ is a Banach space, Boasso proved that for a Moore-Penrose invertible operator $T, T$ is an EP operator if and only there exists an invertible operator $P \in L(X)$ such that $T^{\dagger}=P T$. We generalize this result to the ring case. Moreover, for $a \in R^{\dagger}$, we show that $a \in R^{\mathrm{EP}}$ if and only if there exists a (left) invertible element $v$ such that $a^{\dagger}=v a$. Similarly, for $a \in R^{\oplus}$, then $a \in R^{\mathrm{EP}}$ if and only if there exists a (left) invertible element $s$ such that $a^{\boxplus}=s a$.

In [17], Rakić et al. proved that $a \in R^{\mathrm{EP}}$ if and only if $a \in R^{\dagger} \cap R^{\#}$ with $a^{\dagger}=a^{\boxplus}$. Also, it is proved that $a \in R^{\mathrm{EP}}$ if and only if $a \in R^{\boxplus}$ with $a^{\#}=a^{\boxplus}$. In [14, Theorem 2.1], 
Mosić and Djordjević proved that $a \in R^{\mathrm{EP}}$ if and only if $a \in R^{\#} \cap R^{\dagger}$ with $a^{n} a^{\dagger}=a^{\dagger} a^{n}$ for all choices $n \geqslant 1$. This result also can be found in [7, Theorem 2.4] by Chen. Motivated by $[14,17]$, we will give more new equivalent conditions under which a core invertible element is an EP element. And we define the concept of $n$-EP as a generalization of bi-EP. As a application, we will use $n$-EP property to give an equivalent characterization of the EP elements in $R$.

\section{New characterizations of EP elements by equations}

In this section, we first show that any EP element in $R$ can be characterized by three equations. Let us begin with an auxiliary lemma.

Lemma 2.1. [11, Theorem 7.3] Let $a \in R$. Then $a \in R^{\mathrm{EP}}$ if and only if $a \in R^{\#}$ with $\left(a a^{\#}\right)^{*}=a a^{\#}$.

It is well known that the group inverse of an element in a ring can be characterized by three equations and the Moore-Penrose inverse of an element in a ring can be characterized by four equations. In the following theorem, we show that an EP element in a ring can be described by three equations.

Theorem 2.2. Let $a \in R$. Then $a \in R^{\mathrm{EP}}$ if and only if there exists $x \in R$ such that

$$
(x a)^{*}=x a, \quad x a^{2}=a \text { and } a x^{2}=x .
$$

Proof. Suppose $a \in R^{\mathrm{EP}}$. Let $x=a^{\dagger}=a^{\#}$, then $(x a)^{*}=\left(a^{\dagger} a\right)^{*}=a^{\dagger} a=x a, x a^{2}=$ $a^{\#} a^{2}=a$ and $a x^{2}=a\left(a^{\#}\right)^{2}=a^{\#}=x$. Conversely, if there exists $x \in R$ such that $(x a)^{*}=x a, x a^{2}=a$ and $a x^{2}=x$, then $a\left(x^{2} a\right)=\left(a x^{2}\right) a=x a=x\left(x a^{2}\right)=\left(x^{2} a\right) a$, $a\left(x^{2} a\right) a=(x a) a=x a^{2}=a$, and $\left(x^{2} a\right) a\left(x^{2} a\right)=(x a)\left(x^{2} a\right)=x\left(a x^{2}\right) a=x^{2} a$. These three equalities prove that $a \in R^{\#}, a^{\#}=x^{2} a$, and $a a^{\#}=x a$. By Lemma 2.1, we get $a \in R^{\mathrm{EP}}$.

For an idempotent $p$ in a ring $R$, every $a \in R$ can be written as

$$
a=p a p+p a(1-p)+(1-p) a p+(1-p) a(1-p)
$$

or in the matrix form

$$
a=\left[\begin{array}{ll}
a_{11} & a_{12} \\
a_{21} & a_{22}
\end{array}\right],
$$

where $a_{11}=p a p, a_{12}=p a(1-p), a_{21}=(1-p) a p$ and $a_{22}=(1-p) a(1-p)$.

Let us observe that $p R p$ and $(1-p) R(1-p)$ are subrings whose unities are $p$ and $1-p$, respectively. Also, we notice that if $p=p^{*}$, then the above matrix representation preserves the involution. The term projection will be reserved for a Hermitian idempotent.

Suppose in this paragraph that $a \in R$ is an EP element. If we denote $p=a a^{\dagger}=a^{\dagger} a$, since $a p=p a=a$ and $a^{\dagger} p=p a^{\dagger}=a^{\dagger}$, then the matrix representations of $a$ and $a^{\dagger}$ with respect to the Hermitian idempotent $p$ are

$$
a=\left[\begin{array}{ll}
a & 0 \\
0 & 0
\end{array}\right] \text { and } a^{\dagger}=\left[\begin{array}{ll}
\dagger & 0 \\
0 & 0
\end{array}\right]
$$


respectively.

Recall that a ring $R$ is prime if for any two elements $a$ and $b$ of $R$, arb $=0$ for all $r$ in $R$ implies that either $a=0$ or $b=0$.

In next theorem, the set of elements $x \in R^{\mathrm{EP}}$ satisfying (2.1) is described.

Theorem 2.3. Let $a \in R$. If $a$ is EP, then $\left\{x \in R:(x a)^{*}=x a, x a^{2}=a, a x^{2}=x\right\}=$ $\left\{a^{\dagger}+a a^{\dagger} y\left(1-a a^{\dagger}\right): y \in R\right\}$. Moreover, if $R$ is a prime ring, then $\left\{x \in R:(x a)^{*}=\right.$ $\left.x a, x a^{2}=a, a x^{2}=x\right\}=\left\{a^{\dagger}\right\}$ if and only if $a=0$ or $a$ is invertible.

Proof. Suppose $a$ is an EP element. We use the matrix representations of $a$ and $a^{\dagger}$ with respect to the projection $p=a a^{\dagger}$ given in (2.2). Let $x=\left[\begin{array}{ll}u & v \\ w & z\end{array}\right]$ be the representation of any $x \in R$ with respect to $p$. From $x a^{2}=a$, we get

$$
\left[\begin{array}{ll}
a & 0 \\
0 & 0
\end{array}\right]=\left[\begin{array}{ll}
u & v \\
w & z
\end{array}\right]\left[\begin{array}{cc}
a^{2} & 0 \\
0 & 0
\end{array}\right]=\left[\begin{array}{ll}
u a^{2} & 0 \\
w a^{2} & 0
\end{array}\right]
$$

Since $a$ is EP and $a a^{\dagger}=p=a^{\dagger} a$, then $a$ is invertible in $p R p$ and its inverse is $a^{\dagger}$. Hence from $a=u a^{2}$ and $0=w a^{2}$, we obtain $u=a^{\dagger}$ and $0=w$, respectively. Now, from $x=a x^{2}$ we have

$$
\left[\begin{array}{cc}
a^{\dagger} & v \\
0 & z
\end{array}\right]=\left[\begin{array}{ll}
a & 0 \\
0 & 0
\end{array}\right]\left[\begin{array}{cc}
a^{\dagger} & v \\
0 & z
\end{array}\right] x=\left[\begin{array}{cc}
p & a v \\
0 & 0
\end{array}\right]\left[\begin{array}{cc}
a^{\dagger} & v \\
0 & z
\end{array}\right]=\left[\begin{array}{cc}
a^{\dagger} & v+a v z \\
0 & 0
\end{array}\right],
$$

which implies $z=0$. Therefore,

$$
x=\left[\begin{array}{cc}
a^{\dagger} & v \\
0 & 0
\end{array}\right]=a^{\dagger}+v,
$$

that is $\left\{x \in R:(x a)^{*}=x a, x a^{2}=a, a x^{2}=x\right\} \subseteq\left\{a^{\dagger}+a a^{\dagger} y\left(1-a a^{\dagger}\right): y \in R\right\}$.

Let us prove the opposite inclusion. We have that $a a^{\dagger}=a^{\dagger} a$ since $a$ is EP. Then

$$
\begin{aligned}
& {\left[a^{\dagger}+a a^{\dagger} y\left(1-a a^{\dagger}\right)\right] a=a^{\dagger} a \text { is Hermitian, }} \\
& {\left[a^{\dagger}+a a^{\dagger} y\left(1-a a^{\dagger}\right)\right] a^{2}=a^{\dagger} a^{2}=a,} \\
& a\left[a^{\dagger}+a a^{\dagger} y\left(1-a a^{\dagger}\right)\right]^{2}=\left[a a^{\dagger}+a y\left(1-a a^{\dagger}\right)\right]\left[a^{\dagger}+a a^{\dagger} y\left(1-a a^{\dagger}\right)\right]=a^{\dagger}+a a^{\dagger} y\left(1-a a^{\dagger}\right)
\end{aligned}
$$

Suppose that $R$ is prime ring. If $a=0$, then $\left\{x \in R:(x a)^{*}=x a, x a^{2}=a, a x^{2}=\right.$ $x\}=\{0\}$. If $a$ is invertible, then $\left\{x \in R:(x a)^{*}=x a, x a^{2}=a, a x^{2}=x\right\}=\left\{a^{-1}\right\}$. If $\left\{x \in R:(x a)^{*}=x a, x a^{2}=a, a x^{2}=x\right\}$ is a singleton, then $a a^{\dagger} y\left(1-a a^{\dagger}\right)=0$ for all $y \in R$, by using that $R$ is prime, then $a a^{\dagger}=0$ or $1-a a^{\dagger}=0$. The first of the previous alternatives is equivalent to $a=0$ and the second one (since $a$ is EP) is equivalent to the invertibility of $a$.

We will also use the following notations: $a R=\{a x: x \in R\}, R a=\{x a: x \in R\}$, ${ }^{\circ} a=\{x \in R: x a=0\}, a^{\circ}=\{x \in R: a x=0\}$ and $[a, b]=a b-b a$. The following lemma will be useful in the sequel.

Lemma 2.4. [19] Let $a, b \in R$. Then:

(1) $a R \subseteq b R$ implies ${ }^{\circ} b \subseteq{ }^{\circ} a$ and the converse is valid whenever $b$ is regular; 
(2) $R a \subseteq R b$ implies $b^{\circ} \subseteq a^{\circ}$ and the converse is valid whenever $b$ is regular.

Theorem 2.5. Let $a \in R$. Then the following are equivalent:

(1) $a \in R^{\mathrm{EP}}$;

(2) there exists $x \in R$ such that axa $=a, x R=a R$ and $R x^{*}=R a$;

(3) there exists $x \in R$ such that axa $=a, x R=a R$ and $R x^{*} \subseteq R a$;

(4) there exists $x \in R$ such that $x a x=x, x R=a R$ and $R x^{*}=R a$;

(5) there exists $x \in R$ such that $x a x=x, x R=a R$ and $R a \subseteq R x^{*}$;

(6) there exists $x \in R$ such that axa $=a,{ }^{\circ} x={ }^{\circ} a$ and $\left(x^{*}\right)^{\circ}=a^{\circ}$;

(7) there exists $x \in R$ such that axa $=a,{ }^{\circ} x={ }^{\circ} a$ and $a^{\circ} \subseteq\left(x^{*}\right)^{\circ}$;

(8) there exists $x \in R$ such that $x a x=x,{ }^{\circ} x={ }^{\circ} a$ and $\left(x^{*}\right)^{\circ}=a^{\circ}$;

(9) there exists $x \in R$ such that $x a x=x,{ }^{\circ} x={ }^{\circ} a$ and $\left(x^{*}\right)^{\circ} \subseteq a^{\circ}$.

Proof. (1) $\Rightarrow(2)$ : Let $x=a^{\dagger}=a^{\#}$, then $a x a=a, x=a\left(a^{\#}\right)^{2}, x^{*}=\left(a^{\dagger}\right)^{*}=\left(a^{\dagger} a a^{\dagger}\right)^{*}=$ $\left(a^{\dagger}\right)^{*} a^{\dagger} a$, and $x a^{2}=a^{\#} a^{2}=a=a a^{\dagger} a=a a^{*}\left(a^{\dagger}\right)^{*}=a a^{*} x^{*}$. Thus $x R=a R$ and $R x^{*}=R a$.

$(2) \Rightarrow(3)$ and $(6) \Rightarrow(7)$ are clear.

$(2) \Rightarrow(6)$ and $(3) \Rightarrow(7)$ are obvious by Lemma 2.4 .

$(7) \Rightarrow(1)$ : Suppose there exists $x \in R$ such that $a x a=a,{ }^{\circ} x={ }^{\circ} a$ and $a^{\circ} \subseteq\left(x^{*}\right)^{\circ}$. Since $(1-a x) a=0$, then $1-a x \in{ }^{\circ} a={ }^{\circ} x$, hence $(1-a x) x=0$. Since $a(1-x a)=0$, then $1-x a \in a^{\circ} \subseteq\left(x^{*}\right)^{\circ}$, hence $x^{*}(1-x a)=0$, i.e., $x=(x a)^{*} x$. We get $x a=(x a)^{*} x a$, hence $x a$ is Hermitian. Finally, $x=x a x$ implies $1-x a \in{ }^{\circ} x={ }^{\circ} a$, whence $x a^{2}=a$ Therefore $a \in R^{\mathrm{EP}}$ by Theorem 2.2 .

The implications $(1) \Leftrightarrow(4) \Leftrightarrow(5) \Leftrightarrow(8) \Rightarrow(9)$ are similar to $(1) \Leftrightarrow(2) \Leftrightarrow(3) \Leftrightarrow$ (6) $\Rightarrow(7)$.

$(9) \Rightarrow(1)$ : There exists $x \in R$ such that $x a x=x,{ }^{\circ} x={ }^{\circ} a$ and $\left(x^{*}\right)^{\circ} \subseteq a^{\circ}$. It is obvious that $\left(x^{*}\right)^{\circ} \subseteq a^{\circ}$ is equivalent to ${ }^{\circ} x \subseteq{ }^{\circ}\left(a^{*}\right)$. We have $a=x a^{2}$ since $(1-x a) x=0$ implies $(1-x a) a=0$. Similarly, we have $a^{*}=x a a^{*}$ since $(1-x a) x=0$ implies $(1-x a) a^{*}=0$. Thus $(x a)^{*}=a^{*} x^{*}=x a a^{*} x^{*}=x a(x a)^{*}$, that is $(x a)^{*}=x a$. By $a^{*}=x a a^{*}$ and $(x a)^{*}=x a$, we have $a^{*}=x a a^{*}=(x a)^{*} a^{*}=(a x a)^{*}$, that is $a=a x a$. Hence by ${ }^{\circ} x={ }^{\circ} a$, we have $(1-a x) a=0$ implies $(1-a x) x=0$, which gives $x=a x^{2}$. Therefore $a \in R^{\mathrm{EP}}$ by Theorem 2.2 .

Theorem 2.6. Let $a \in R^{\mathrm{EP}}$ and denote $p=a a^{\dagger}$. Then

(1) $\{x \in R: a x a=a, x \in a R\}=\left\{a^{\dagger}+p y(1-p): y \in R\right\}$;

(2) $\{x \in R: x a x=x, x R=a R\}=\left\{a^{\dagger}+p z(1-p): z \in R\right\}$;

(3) $\left\{x \in R: a x a=a,{ }^{\circ} a \subseteq{ }^{\circ} x\right\}=\left\{a^{\dagger}+p y^{\prime}(1-p): y^{\prime} \in R\right\}$;

(4) $\left\{x \in R: x a x=x,{ }^{\circ} a={ }^{\circ} x\right\}=\left\{a^{\dagger}+p z^{\prime}(1-p): z^{\prime} \in R\right\}$. 
Furthermore, if $R$ is prime, then any of the above subsets is a singleton if and only if $a=0$ or a is invertible.

Proof. We use the matrix representations of $a$ and $a^{\dagger}$ with respect to the projection $p=a a^{\dagger}$ given in (2.2). Let $x=\left[\begin{array}{ll}u & v \\ w & z\end{array}\right]$ be the representation of any $x$ with respect to $p$.

(1) Let $x$ satisfy $a x a=a$ and $x \in a R$. From $a x a=a$, we have

$$
\left[\begin{array}{ll}
a & 0 \\
0 & 0
\end{array}\right]=\left[\begin{array}{ll}
a & 0 \\
0 & 0
\end{array}\right]\left[\begin{array}{ll}
u & v \\
w & z
\end{array}\right]\left[\begin{array}{ll}
a & 0 \\
0 & 0
\end{array}\right]=\left[\begin{array}{cc}
a u & a v \\
0 & 0
\end{array}\right]\left[\begin{array}{cc}
a & 0 \\
0 & 0
\end{array}\right]=\left[\begin{array}{cc}
a u a & 0 \\
0 & 0
\end{array}\right] .
$$

Since $a \in R^{\mathrm{EP}}$, we have that $a$ is invertible in $p R p$ and its inverse is $a^{\dagger}$. Hence $a=a u a$ gives $u=a^{\dagger}$. Since $x \in a R$, we can write

$$
\left[\begin{array}{ll}
u & v \\
w & z
\end{array}\right]=\left[\begin{array}{ll}
a & 0 \\
0 & 0
\end{array}\right]\left[\begin{array}{ll}
\xi_{1} & \xi_{2} \\
\xi_{3} & \xi_{4}
\end{array}\right]=\left[\begin{array}{cc}
a \xi_{1} & a \xi_{2} \\
0 & 0
\end{array}\right]
$$

Therefore, $w=z=0$. Hence

$$
x=\left[\begin{array}{cc}
a^{\dagger} & v \\
0 & 0
\end{array}\right]=a^{\dagger}+p x(1-p) \in\left\{a^{\dagger}+p y(1-p): y \in R\right\} .
$$

The opposite inclusion is trivial.

(2) Let $x \in R$ satisfy $x a x=x$ and $x R=a R$. Since $x \in a R$, by the proof of (1), we have $w=z=0$. Now, since $a \in x R$, we can write

$$
\left[\begin{array}{ll}
a & 0 \\
0 & 0
\end{array}\right]=\left[\begin{array}{ll}
u & v \\
0 & 0
\end{array}\right]\left[\begin{array}{ll}
\delta_{1} & \delta_{2} \\
\delta_{3} & \delta_{4}
\end{array}\right]
$$

which implies

$$
a=u \delta_{1}+v \delta_{3}, \quad 0=u \delta_{2}+v \delta_{4} .
$$

Now, we use $x a x=x:$

$$
\left[\begin{array}{ll}
u & v \\
0 & 0
\end{array}\right]=\left[\begin{array}{ll}
u & v \\
0 & 0
\end{array}\right]\left[\begin{array}{ll}
a & 0 \\
0 & 0
\end{array}\right]\left[\begin{array}{cc}
u & v \\
0 & 0
\end{array}\right]=\left[\begin{array}{cc}
u a & 0 \\
0 & 0
\end{array}\right]\left[\begin{array}{cc}
u & v \\
0 & 0
\end{array}\right]=\left[\begin{array}{cc}
u a u & u a v \\
0 & 0
\end{array}\right] .
$$

Therefore

$$
u=u a u, \quad v=u a v .
$$

Post-multiply the first equality of (2.4) by $\delta_{1}$ and the second equality of (2.4) by $\delta_{3}$ to obtain

$$
u \delta_{1}=u a u \delta_{1}, \quad v \delta_{3}=u a v \delta_{3} .
$$

From (2.3)

$$
a=u \delta_{1}+v \delta_{3}=u a u \delta_{1}+u a v \delta_{3}=u a\left(u \delta_{1}+v \delta_{3}\right)=u a^{2} .
$$

We get $u=a^{\dagger}$ because $a$ is invertible in $p R p$ and its inverse is $a^{\dagger}$. Therefore

$$
x=\left[\begin{array}{cc}
a^{\dagger} & v \\
0 & 0
\end{array}\right]=a^{\dagger}+v=a^{\dagger}+p x(1-p) \in\left\{a^{\dagger}+p z(1-p): z \in R\right\} .
$$


For the opposite inclusion, it is easy to check that $\left[a^{\dagger}+p z(1-p)\right] a\left[a^{\dagger}+p z(1-p)\right]=$ $a^{\dagger}+p z(1-p)$ and $a^{\dagger}+p z(1-p) \in a R$ in view of $a \in R^{\mathrm{EP}}$. From $a=\left[a^{\dagger}+p z(1-p)\right] a^{2}$, we deduce that $a \in\left[a^{\dagger}+p z(1-p)\right] R$.

The proof of statements (3) and (4) follows from (1) and (2), respectively, since by Lemma 2.4, we obtain that $x \in a R$ is equivalent to ${ }^{\circ} a \subseteq{ }^{\circ} x$ and $x R=a R$ is equivalent to ${ }^{\circ} a={ }^{\circ} x$, respectively.

The proof of the last affirmation of this theorem has the same proof as the corresponding part of Theorem 2.3.

By considering that $a$ is EP if and only if $a^{*}$ is EP and having in mind Theorem 2.2, Theorem 2.3, Theorem 2.5 and Theorem 2.6, we get the following four theorems.

Theorem 2.7. Let $a \in R$. Then $a \in R^{\mathrm{EP}}$ if and only if there exists $y \in R$ such that

$$
(a y)^{*}=a y, \quad a^{2} y=a \text { and } y^{2} a=y .
$$

Theorem 2.8. Let $a \in R$. If $a$ is $E P$, then $\left\{y \in R:(a y)^{*}=a y, a^{2} y=a, y^{2} a=y\right\}=$ $\left\{a^{\dagger}+\left(1-a a^{\dagger}\right) x a a^{\dagger}: x \in R\right\}$. Moreover, if $R$ is a prime ring, then $\left\{y \in R:(a y)^{*}=\right.$ $\left.a y, a^{2} y=a, y^{2} a=y\right\}=\left\{a^{\dagger}\right\}$ if and only if $a=0$ or $a$ is invertible.

Theorem 2.9. Let $a \in R$. Then the following are equivalent:

(1) $a \in R^{\mathrm{EP}}$;

(2) there exists $y \in R$ such that aya $=a, R y=R a$ and $y^{*} R=a R$;

(3) there exists $y \in R$ such that aya $=a, R y=R a$ and $y^{*} R \subseteq a R$;

(4) there exists $y \in R$ such that yay $=y, R y=R a$ and $y^{*} R=a R$;

(5) there exists $y \in R$ such that yay $=y, R y=R a$ and $a R \subseteq y^{*} R$;

(6) there exists $y \in R$ such that aya $=a, y^{\circ}=a^{\circ}$ and ${ }^{\circ}\left(y^{*}\right)={ }^{\circ} a$;

(7) there exists $y \in R$ such that aya $=a, y^{\circ}=a^{\circ}$ and ${ }^{\circ} a \subseteq^{\circ}\left(y^{*}\right)$;

(8) there exists $y \in R$ such that yay $=y, y^{\circ}=a^{\circ}$ and ${ }^{\circ}\left(y^{*}\right)={ }^{\circ} a$;

(9) there exists $y \in R$ such that yay $=y, y^{\circ}=a^{\circ}$ and ${ }^{\circ}\left(y^{*}\right) \subseteq{ }^{\circ} a$.

Theorem 2.10. Let $a \in R^{E P}$ and denote $p=a a^{\dagger}$. Then

(1) $\{y \in R: a y a=a, y \in R a\}=\left\{a^{\dagger}+(1-p) x p: x \in R\right\}$;

(2) (2) $\{y \in R:$ yay $=y, R y=R a\}=\left\{a^{\dagger}+(1-p) z p: z \in R\right\}$;

(3) $\left\{y \in R: a y a=a, a^{\circ} \subseteq y^{\circ}\right\}=\left\{a^{\dagger}+(1-p) x^{\prime} p: x^{\prime} \in R\right\}$;

(4) $\left\{y \in R:\right.$ yay $\left.=y, a^{\circ}=y^{\circ}\right\}=\left\{a^{\dagger}+(1-p) z^{\prime} p: z^{\prime} \in R\right\}$.

Furthermore, if $R$ is prime, then any of the above subsets is a singleton if and only if $a=0$ or a is invertible. 
We will characterize when $a \in R$ is EP by another subset of three equations.

Theorem 2.11. Let $a \in R$. Then $a \in R^{\mathrm{EP}}$ if and only if there exists $x \in R$ such that

$$
a^{2} x=a, \quad a x=x a \text { and }(a x)^{*}=a x .
$$

Proof. If $a$ is EP, by taking $x=a^{\dagger}=a^{\#}$, we get (2.5). Conversely, assume that exists $x \in R$ such that (2.5) is satisfied. We shall show that $a \in R^{\mathrm{EP}}$ and $a^{\#}=a x^{2}$. Since $a x=x a$, we get $a\left(a x^{2}\right)=\left(a x^{2}\right) a$, but in addition, $a\left(a x^{2}\right)=\left(a^{2} x\right) x=a x$, which leads to $a\left(a x^{2}\right) a=a^{2} x=a$ and $\left(a x^{2}\right) a\left(a x^{2}\right)=\left(a x^{2}\right) a x=\left(a^{2} x\right) x^{2}=a x^{2}$. Since $a a^{\#}=a^{2} x^{2}=a x$ is Hermitian, the conclusion follows from Lemma 2.1.

We have seen that if $a \in R$ is EP, then $\left\{x \in R: a^{2} x=a, a x=x a,(a x)^{*}=a x\right\}$ is not empty. In next theorem we describe this last set.

Theorem 2.12. Let $a \in R$. If $a$ is $E P$, then $\left\{x \in R: a^{2} x=a, a x=x a,(a x)^{*}=a x\right\}=$ $\left\{a^{\dagger}+\left(1-a a^{\dagger}\right) y\left(1-a a^{\dagger}\right): y \in R\right\}$. Moreover, if $R$ is a prime ring, then $\left\{x \in R: a^{2} x=\right.$ $\left.a, a x=x a,(a x)^{*}=a x\right\}=\left\{a^{\dagger}\right\}$ if and only if $a$ is invertible.

Proof. Suppose that $a$ is an EP element. We use the matrix representations of $a$ and $a^{\dagger}$ with respect to the projection $p=a a^{\dagger}$ given in (2.2). Let $x=\left[\begin{array}{ll}u & v \\ w & z\end{array}\right]$ be the representation of any $x \in R$ with respect to $p$.

Let $x \in R$ satisfy $a^{2} x=a, a x=x a$ and $(a x)^{*}=a x$. From $a^{2} x=a$, we get

$$
\left[\begin{array}{ll}
a & 0 \\
0 & 0
\end{array}\right]^{2}\left[\begin{array}{ll}
u & v \\
w & z
\end{array}\right]=\left[\begin{array}{ll}
a & 0 \\
0 & 0
\end{array}\right]
$$

which leads to $a^{2} u=a$ and $a^{2} v=0$. Since $a$ is EP and $a a^{\dagger}=p=a^{\dagger} a$, then $a$ is invertible in $p R p$ and its inverse is $a^{\dagger}$. Hence from $a=a^{2} u$ and $0=a^{2} v$, we obtain $u=a^{\dagger}$ and $0=v$, respectively. Now, from $a x=x a$ we have

$$
\left[\begin{array}{ll}
a & 0 \\
0 & 0
\end{array}\right]\left[\begin{array}{ll}
a^{\dagger} & 0 \\
w & z
\end{array}\right]=\left[\begin{array}{ll}
a^{\dagger} & 0 \\
w & z
\end{array}\right]\left[\begin{array}{ll}
a & 0 \\
0 & z
\end{array}\right]
$$

which implies $0=w a$, and taking into account that $a$ is invertible in $p R p$, we have $0=w$. Therefore,

$$
x=\left[\begin{array}{cc}
a^{\dagger} & 0 \\
0 & z
\end{array}\right]=a^{\dagger}+z,
$$

that is $\left\{x \in R: a^{2} x=a, a x=x a,(a x)^{*}=a x\right\} \subseteq\left\{a^{\dagger}+\left(1-a a^{\dagger}\right) y\left(1-a a^{\dagger}\right): y \in R\right\}$.

Let us prove the opposite inclusion. We have $a a^{\dagger}=a^{\dagger} a$ since $a$ is EP. Now,

$$
\begin{aligned}
& a\left[a^{\dagger}+\left(1-a a^{\dagger}\right) y\left(1-a a^{\dagger}\right)\right]=a a^{\dagger} \text { is Hermitian, } \\
& a^{2}\left[a^{\dagger}+\left(1-a a^{\dagger}\right) y\left(1-a a^{\dagger}\right)\right]=a^{2} a^{\dagger}=a, \\
& a\left[a^{\dagger}+\left(1-a a^{\dagger}\right) y\left(1-a a^{\dagger}\right)\right]=a a^{\dagger}=a^{\dagger} a=\left[a^{\dagger}+\left(1-a a^{\dagger}\right) y\left(1-a a^{\dagger}\right)\right] a .
\end{aligned}
$$

Suppose that $R$ is a prime ring. If $a$ is invertible, then $\left\{x \in R: a^{2} x=a, a x=\right.$ $\left.x a,(a x)^{*}=a x\right\}=\left\{a^{-1}\right\}$. If $\left\{x \in R: a^{2} x=a, a x=x a,(a x)^{*}=a x\right\}$ is a singleton, then $\left(1-a a^{\dagger}\right) y\left(1-a a^{\dagger}\right)=0$ for all $y \in R$. By using that $R$ is prime, we get $1-a a^{\dagger}=0$, which (since $a$ is $\mathrm{EP}$ ) is equivalent to the invertibility of $a$. 
Theorem 2.13. Let $a \in R$. Then the following are equivalent:

(1) $a \in R^{\mathrm{EP}}$;

$(2)^{\circ}\left(a^{2}\right) \subseteq{ }^{\circ} a$ and there exists $x \in R$ such that $x a^{2}=a$ and $(x a)^{*}=x a$;

(3) $\left(a^{2}\right)^{\circ} \subseteq a^{\circ}$ and there exists $x \in R$ such that $a^{2} x=a$ and $(a x)^{*}=a x$.

Furthermore, under these equivalences one has that the set of elements $x$ satisfying (2) is $\left\{a^{\dagger}+y\left(1-a a^{\dagger}\right): y \in R\right\}$ and the set of elements $x$ satisfying $(3)$ is $\left\{a^{\dagger}+\left(1-a a^{\dagger}\right) z: z \in R\right\}$. If $R$ is prime and $a$ is EP, then the sets of $x$ satisfying (2) or (3) is $\left\{a^{\dagger}\right\}$.

Proof. (1) $\Rightarrow(2)$ : The inclusion ${ }^{\circ}\left(a^{2}\right) \subseteq{ }^{\circ} a$ is evident from $a \in R^{\#}$. For the remaining, it is sufficient to take $x=a^{\dagger}=a^{\#}$.

$(2) \Rightarrow(1)$ : Since $(a x-1) a^{2}=a\left(x a^{2}\right)-a^{2}=a^{2}-a^{2}=0$, we get $a x-1 \in{ }^{\circ}\left(a^{2}\right) \subseteq{ }^{\circ} a$, hence $a x a=a$. From

$$
a x^{2} a^{2}=a x\left(x a^{2}\right)=a x a=a=x a^{2}
$$

we get $a x^{2}-x \in{ }^{\circ}\left(a^{2}\right) \subseteq{ }^{\circ} a$, hence $a x^{2} a=x a$. Now, we prove $a^{\#}=x^{2} a$ by the definition of the group inverse,

$$
\begin{gathered}
a\left(x^{2} a\right)=a x^{2} a=x a, \quad\left(x^{2} a\right) a=x\left(x a^{2}\right)=x a ; \\
a\left(x^{2} a\right) a=x a^{2}=a ; \quad\left(x^{2} a\right) a\left(x^{2} a\right)=x\left(x a^{2}\right) x^{2} a=x a x^{2} a=x\left(a x^{2} a\right)=x^{2} a .
\end{gathered}
$$

Now, $a^{\#}=x^{2} a$ and $a a^{\#}=a x^{2} a=x a$ is Hermitian. Hence (1) follows from Lemma 2.1.

The proof of $(1) \Leftrightarrow(3)$ is similar to the proof of $(1) \Leftrightarrow(2)$.

Now, let us prove the last part of the theorem. Recall that $a$ is EP. If $x \in R$ satisfies $x a^{2}=a$, then $\left(x-a^{\dagger}\right) a a^{\dagger}=x a a^{\dagger}-a^{\dagger}=x a^{2}\left(a^{\dagger}\right)^{2}-a^{\dagger}=a\left(a^{\dagger}\right)^{2}-a^{\dagger}=0$. Hence $x-a^{\dagger}=\left(x-a^{\dagger}\right)\left(1-a a^{\dagger}\right)$, which yields $x \in\left\{a^{\dagger}+y\left(1-a a^{\dagger}\right): y \in R\right\}$. Reciprocally, it is evident that for any $y \in R$ one has that $\left[a^{\dagger}+y\left(1-a a^{\dagger}\right)\right] a^{2}=a$ and $\left[a^{\dagger}+y\left(1-a a^{\dagger}\right)\right] a$ is Hermitian. The affirmation concerning the primality of $R$ has the same proof as the corresponding in previous Theorem 2.12.

\section{When a core invertible element is an EP element}

Any EP element is core invertible, but when a core invertible element is EP? In this section we answer this question. Let us start this section with a lemma.

Lemma 3.1. [17, Theorem 2.14] An element $a \in R$ is core invertible if and only if there exists $x \in R$ such that

$$
a x a=a, \quad x a x=x, \quad(a x)^{*}=a x, \quad x a^{2}=a \text { and } a x^{2}=x .
$$

Under this equivalence, one has that $x=a^{\boxplus}$.

Let us recall the following result.

Theorem 3.2. Let $a \in R$. 
(1) [5, Proposition 8.24] a is group invertible if and only if exists an idempotent $p \in R$ such that $a p=p a=0$ and $a+p$ is invertible. Under this equivalence, we have $p=1-a a^{\#}$ and $a^{\#}=(a+p)^{-1}-p$.

(2) $[4$, Theorem 2.1] $a$ is EP if and only if exists a projection $p \in R$ such that ap $=$ $p a=0$ and $a+p$ is invertible. Under this equivalence, we have $p=1-a a^{\dagger}$ and $a^{\dagger}=(a+p)^{-1}-p$.

In fact, the second item of previous result was stated for unital $C^{*}$-algebras, but as one can easily check, its proof remains valid for unital rings with an involution. We give a similar characterization of the core invertibility.

Theorem 3.3. Let $a \in R$. The following affirmations are equivalent:

(1) a is core invertible.

(2) Exists a projection $p$ such that $p a=0$ and $a(1-p)$ is invertible in the subring $(1-p) R(1-p)$.

(3) Exists a projection $p$ such that $p a=0$ and $a(1-p)+p$ is invertible.

(4) Exists a projection $p$ such that $p a=0$ and $a+p$ is invertible.

Under this equivalence, one has this projection $p$ is unique and $p=1-a a^{\boxplus}$. In addition,

$$
(a(1-p))_{(1-p) R(1-p)}^{-1}=a^{\oplus}, \quad(a(1-p)+p)^{-1}=p+a^{\oplus}, \quad(a+p)^{-1}=p-a^{\oplus} a p+a^{\oplus} .
$$

Proof. (1) $\Rightarrow(2)$ : Let us represent $a$ with respect to the projection $p=1-a a^{\boxplus}$. We use the notation $\bar{p}=1-p=a a^{\boxplus}$. Observe that from Lemma 3.1 we have $p a=\left(1-a a^{\boxplus}\right) a=0$ and $a^{\boxplus} p=a^{\boxplus}\left(1-a a^{\boxplus}\right)=0$. Therefore,

$$
a=\left[\begin{array}{cc}
p a p & p a \bar{p} \\
\bar{p} a p & \bar{p} a \bar{p}
\end{array}\right]=\left[\begin{array}{cc}
0 & 0 \\
a p & a \bar{p}
\end{array}\right] \quad \text { and } \quad a^{\oplus}=\left[\begin{array}{cc}
p a^{\oplus} p & p a^{\oplus} \bar{p} \\
\bar{p} a^{\oplus} p & \bar{p} a^{\oplus} \bar{p}
\end{array}\right]=\left[\begin{array}{cc}
0 & p a^{\boxplus} \\
0 & \bar{p} a^{\oplus}
\end{array}\right] .
$$

Now, $a\left(a^{\boxplus}\right)^{2}=a^{\boxplus}$ leads to $\bar{p} a^{\boxplus}=a^{\boxplus}$ and $p a^{\boxplus}=0$. Hence

$$
a^{\oplus}=\left[\begin{array}{cc}
0 & 0 \\
0 & a^{\oplus}
\end{array}\right] \in \bar{p} R \bar{p} .
$$

From $a^{\oplus} a^{2}=a$ we have $a^{\boxplus} a^{2} a a^{\oplus}=a a^{\oplus}$, i.e., $a^{\oplus a \bar{p}}=\bar{p}$. Furthermore, $a \bar{p} a^{\oplus}=a a^{\oplus}=\bar{p}$. Therefore, $a \bar{p} \in \bar{p} R \bar{p}$ is invertible in the subring $\bar{p} R \bar{p}$ and its inverse is $a^{\boxplus}$.

$(2) \Leftrightarrow(3) \Leftrightarrow(4)$ : Let $p \in R$ be a projection such that $p a=0$. The representation of $a$ with respect to $p$ is the same as in (3.1). Now we have

$$
a(1-p)=\left[\begin{array}{cc}
0 & 0 \\
0 & a(1-p)
\end{array}\right], \quad a(1-p)+p=\left[\begin{array}{cc}
p & 0 \\
0 & a(1-p)
\end{array}\right], \quad a+p=\left[\begin{array}{cc}
p & 0 \\
a p & a(1-p)
\end{array}\right] .
$$

Taking into account that $p$ is invertible in the subring $p R p$ (in fact, $p$ is the unity), evidently we have that $a(1-p) \in((1-p) R(1-p))^{-1} \Leftrightarrow a(1-p)+p \in R^{-1} \Leftrightarrow a+p \in R^{-1}$. 
$(2) \Rightarrow(1)$ : Let $x \in(1-p) R(1-p)$ be the inverse of $a(1-p)$ in $(1-p) R(1-p)$. This means that $a(1-p) x=x a(1-p)=1-p$. Observe that $x \in(1-p) R(1-p)$ implies $(1-p) x=x(1-p)=x$, and therefore, $a x=x a(1-p)=1-p$. We will prove that $x=a^{\boxplus}$ by using Lemma 3.1. Let us recall that we can use $p a=0$ and $(1-p) a=a$ by hypothesis.

$$
\begin{gathered}
a x a=(a x) a=(1-p) a=a . \\
x a x=x(a x)=x(1-p)=x . \\
a x=1-p \text { is Hermitian. } \\
x a^{2}=x a(1-p) a=(1-p) a=a . \\
a x^{2}=(a x) x=(1-p) x=x .
\end{gathered}
$$

Now, we shall prove the uniqueness. Assume that $q$ is another projection such that $q a=0$ and $a(1-q)$ is invertible in $(1-q) R(1-q)$. By the proof of $(2) \Rightarrow(1)$ we get that the inverse of $a(1-q)$ in $(1-q) R(1-q)$ is $a^{\boxplus}$, in particular $a^{\boxplus} \in(1-q) R(1-q)$ and $a(1-q) a^{\boxplus}=1-q$. By using also Lemma 3.1 we get

$$
(1-q) a a^{\oplus}=a(1-q) a^{\oplus} a a^{\boxplus}=a(1-q) a^{\oplus}=1-q .
$$

Since $a^{\boxplus} \in(1-q) R(1-q)$, exists $u \in R$ such that $a^{\boxplus}=u(1-q)$. Now,

$$
a a^{\boxplus}(1-q)=a u(1-q)^{2}=a u(1-q)=a a^{\boxplus} .
$$

Apply involution and use Lemma 3.1 in this last equality to get $(1-q) a a^{\boxplus}=a a^{\boxplus}$. From this last equality and (3.2) we obtain $a a^{\boxplus}=1-q$. In other words, we have proved the uniqueness of such $q$.

The expresion of ()

Lemma 3.4. [17, Theorem 3.1] Let $a \in R$. Then the following are equivalent:

(1) $a \in R^{\mathrm{EP}}$;

(2) $a \in R^{\dagger}$ and $\left[a, a^{\dagger}\right]=0$;

(3) $a \in R^{\boxplus}$ and $\left[a, a^{\boxplus}\right]=0$;

(4) $a \in R^{\boxplus}$ and $a^{\#}=a^{\oplus}$;

(5) $a \in R^{\dagger} \cap R^{\#}$ and $a^{\dagger}=a^{\boxplus}$.

Lemma 3.5. [17, Theorem 2.18] Let $a \in R^{\oplus}$. Then $a^{\boxplus} \in R^{\mathrm{EP}}$ and $\left(a^{\boxplus}\right)^{\boxplus}=\left(a^{\boxplus}\right)^{\dagger}=$ $\left(a^{\boxplus}\right)^{\#}=a^{2} a^{\boxplus}$. Moreover, if $a \in R^{\dagger}$, then $\left(a^{\dagger}\right)^{\boxplus}=\left(a^{\boxplus} a\right)^{*} a$.

Lemma 3.6. [16] Let $a \in R$. Then the following are equivalent:

(1) $a \in R^{\mathrm{EP}}$;

(2) $a \in R^{\#}$ and $a R=a^{*} R$;

(3) $a \in R^{\#}$ and $R a=R a^{*}$.

In the following theorem, we show that the equality $a R=a^{*} R$ in Lemma 3.6 can be replaced by weaker inclusions $a R \subseteq a^{*} R$ or $a^{*} R \subseteq a R$. 
Theorem 3.7. Let $a \in R$. Then the following are equivalent:

(1) $a \in R^{\mathrm{EP}}$;

(2) $a \in R^{\#}$ and $a R \subseteq a^{*} R$;

(3) $a \in R^{\#}$ and $R a \subseteq R a^{*}$;

(4) $a \in R^{\#}$ and $a^{*} R \subseteq a R$;

(5) $a \in R^{\#}$ and $R a^{*} \subseteq R a$.

Proof. (1) $\Rightarrow(2)-(5)$ is obvious by Lemma 3.6.

$(2) \Rightarrow(1)$ : By $a R \subseteq a^{*} R$, we have $a=a^{*} r$ for some $r \in R$, then $a=\left(a a^{\#} a\right)^{*} r=$ $\left(a^{\#} a\right)^{*} a^{*} r=\left(a^{\#} a\right)^{*} a$. Thus $a^{\#} a=a a^{\#}=\left(a^{\#} a\right)^{*} a a^{\#}=\left(a^{\#} a\right)^{*} a^{\#} a$, which gives $\left(a^{\#} a\right)^{*}=$ $a^{\#} a$. Therefore $a \in R^{E P}$ by the definition of EP element.

$(3)-(5) \Rightarrow(1)$ is similar to $(2) \Rightarrow(1)$.

Theorem 3.8. Let $a \in R$. Then the following are equivalent:

(1) $a \in R^{\mathrm{EP}}$;

(2) $a \in R^{\oplus}$ and $\left(a^{\oplus} a\right)^{*}=a^{\oplus} a$;

(3) $a \in R^{\oplus}$ and $\left(a^{\oplus}\right)^{\oplus}=a$;

(4) $a \in R^{\boxplus}$ and $\left(a^{\oplus}\right)^{\dagger}=a$;

(5) $a \in R^{\oplus}$ and $\left(a^{\boxplus}\right)^{\#}=a$;

(6) $a \in R^{\dagger} \cap R^{\#}$ and $\left(a^{\dagger}\right)^{\oplus}=a$;

(7) $a \in R^{\dagger} \cap R^{\#}$ and $\left(a^{\dagger}\right)^{\boxplus}=\left(a^{\boxplus}\right)^{\dagger}$;

(8) $a \in R^{\oplus}$ and $a p=0$, where $p=1-a a^{\oplus}$.

Proof. (1) $\Leftrightarrow(2)$ : Suppose $a \in R^{\mathrm{EP}}$. Then by Lemma 3.4, we have $a^{\dagger}=a^{\oplus}$. Thus $\left(a^{\dagger} a\right)^{*}=a^{\dagger} a$ implies $\left(a^{\boxplus} a\right)^{*}=a^{\boxplus} a$. Conversely, suppose $a \in R^{\oplus}$ and $\left(a^{\boxplus} a\right)^{*}=a^{\oplus} a$. By Lemma 3.1, we have $a a^{\boxplus} a=a, a^{\boxplus} a a^{\boxplus}=a^{\boxplus}$ and $\left(a a^{\boxplus}\right)^{*}=a a^{\boxplus}$. Thus by the definition of Moore-Penrose inverse, we have $a^{\dagger}=a^{\boxplus}$. Hence by Lemma 3.4, we have $a \in R^{\mathrm{EP}}$.

$(1) \Leftrightarrow(3)$ : Suppose $a \in R^{\mathrm{EP}}$. Then by Lemma 3.4, we have $\left[a, a^{\oplus}\right]=0$. By Lemma 3.5, we have $\left(a^{\boxplus}\right)^{\oplus}=a^{2} a^{\boxplus}$. Thus $\left(a^{\boxplus}\right)^{\oplus}=a^{2} a^{\boxplus}=a\left(a a^{\boxplus}\right)=a\left(a^{\oplus} a\right)=a$. Conversely, suppose $\left(a^{\boxplus}\right)^{\boxplus}=a$. By Lemma 3.5, we have $\left(a^{\boxplus}\right) \oplus=a^{2} a^{\boxplus}$. Then $a=a^{2} a^{\boxplus}$. Thus

$$
a^{\oplus} a=a^{\oplus} a^{2} a^{\oplus}=a a^{\oplus} .
$$

Therefore $a \in R^{\mathrm{EP}}$ by Lemma 3.4.

$(3) \Leftrightarrow(4) \Leftrightarrow(5)$ is clear by Lemma 3.5 .

$(1) \Rightarrow(6)$ : By Lemma 3.4 and Lemma 3.5, we have $\left(a^{\dagger}\right)^{\oplus}=\left(a^{\oplus} a\right)^{*} a=\left(a^{\dagger} a\right)^{*} a=$ $a^{\dagger} a^{2}=a^{\#} a^{2}=a$.

$(6) \Rightarrow(7)$ : Suppose that $a \in R^{\oplus}$ and $\left(a^{\dagger}\right)^{\oplus}=a$. Then by Lemma 3.1, we have $a^{\dagger} a^{2}=a$ and $a\left(a^{\dagger}\right)^{2}=a^{\dagger}$. Thus $a^{\dagger} a=a\left(a^{\dagger}\right)^{2} a=a^{\dagger} a^{2} a^{\dagger}=a a^{\dagger}$. By Lemma 3.4, we have $a \in R^{\mathrm{EP}}$. Then $a^{\boxplus}=a^{\#}$. By Lemma 3.5, we have $\left(a^{\boxplus}\right)^{\dagger}=a^{2} a^{\boxplus}=a^{2} a^{\#}=a$. Thus by $\left(a^{\dagger}\right)^{\oplus}=a$, we have $\left(a^{\dagger}\right)^{\boxplus}=\left(a^{\boxplus}\right)^{\dagger}$.

$(7) \Rightarrow(1)$ : Suppose that $a \in R^{\boxplus}$ and $\left(a^{\dagger}\right)^{\boxplus}=\left(a^{\boxplus}\right)^{\dagger}$. Then by Lemma 3.5, we have $\left(a^{\boxplus}\right)^{\dagger}=a^{2} a^{\boxplus}$ and $\left(a^{\dagger}\right)^{\boxplus}=\left(a^{\boxplus} a\right)^{*} a$. Thus by $\left(a^{\dagger}\right)^{\boxplus}=\left(a^{\boxplus}\right)^{\dagger}$, we have

$$
a^{2} a^{\oplus}=\left(a^{\oplus} a\right)^{*} a .
$$

Taking involution on (3.3), we have $a^{*} a^{\boxplus} a=\left(a a^{\boxplus}\right)^{*} a^{*}=a a^{\boxplus} a^{*}$. Thus $a^{\boxplus} a=\left(a^{\boxplus}\right)^{*} a^{*} a^{\boxplus} a=$ $\left(a^{\boxplus}\right)^{*} a a^{\boxplus} a^{*}=a a^{\boxplus}$. That is $\left[a, a^{\boxplus}\right]=0$, therefore $a \in R^{\mathrm{EP}}$ by Lemma 3.4. 
$(8) \Leftrightarrow(1)$ : If $a$ is an EP element, then $a p=0$ is clear by $a a^{\oplus}=a^{\oplus} a$. Conversely, assume that (8) holds. Then $a=a^{2} a^{\boxplus}=a\left(a a^{\boxplus}\right)^{*}=a\left(a^{\boxplus}\right)^{*} a^{*} \in R a^{*}$. As a core invertible element is group invertible, thus $a \in R^{\mathrm{EP}}$ by Theorem 3.7.

Example 3.9. If $a \in R$ is core invertible element and there exists a projection $p \in R$ such that $a p=0$, we could not get that $a \in R^{\mathrm{EP}}$. Considering the following counterexample. Let $R$ be the ring of all $2 \times 2$ matrices over in field $\mathbb{F}$ with transpose as involution. Let $A=\left[\begin{array}{ll}0 & 1 \\ 0 & 1\end{array}\right], P=\left[\begin{array}{ll}1 & 0 \\ 0 & 0\end{array}\right]$. Then $A^{\#}=\left[\begin{array}{ll}0 & 1 \\ 0 & 1\end{array}\right], A^{\boxplus}=\left[\begin{array}{cc}\frac{1}{2} & \frac{1}{2} \\ \frac{1}{2} & \frac{1}{2}\end{array}\right], A P=\left[\begin{array}{ll}0 & 0 \\ 0 & 0\end{array}\right]$ and $P^{2}=P=P^{*}$. But $A^{\#} \neq A^{\oplus}$, therefore $A$ is not EP.

Theorem 3.10. Let $a \in R$. Then the following are equivalent:

(1) $a \in R^{\mathrm{EP}}$;

(2) $a \in R^{\oplus}$ and $a R \subseteq a^{*} R$;

(3) $a \in R^{\oplus}$ and $\left[a^{\oplus},\left(a^{\oplus} a\right)^{*} a\right]=0$.

Proof. (1) $\Leftrightarrow(2)$ is easy to see that by Lemma 3.1 and Theorem 3.7.

$(1) \Rightarrow(3)$ : Suppose $a$ is EP, then $a \in R^{\boxplus}$ and $a^{\#}=a^{\dagger}=a^{\boxplus}$. Thus $\left[a^{\boxplus},\left(a^{\boxplus} a\right)^{*} a\right]=$ $\left[a^{\#},\left(a^{\dagger} a\right)^{*} a\right]=\left[a^{\#}, a^{\dagger} a^{2}\right]=\left[a^{\#}, a^{\#} a^{2}\right]=\left[a^{\#}, a\right]=0$.

$(3) \Rightarrow(2)$ : Suppose $\left[a^{\boxplus},\left(a^{\oplus} a\right)^{*} a\right]=0$, then

$$
a^{\oplus}\left(a^{\oplus} a\right)^{*} a=\left(a^{\oplus} a\right)^{*} a a^{\boxplus} .
$$

Taking involution $*$ on (3.4), we can get $a^{*} a^{\boxplus} a\left(a^{\boxplus}\right)^{*}=\left(a^{\boxplus}\right)^{*} a^{*} a^{\boxplus} a$, which gives

$$
a^{*} a^{\boxplus} a\left(a^{\boxplus}\right)^{*}=\left(a^{\boxplus}\right)^{*} a^{*} a^{\oplus} a=\left(a a^{\boxplus}\right)^{*} a^{\boxplus} a=a\left(a^{\boxplus}\right)^{2} a=a^{\boxplus} a .
$$

Therefore $a^{*} a^{\boxplus} a\left(a^{\boxplus}\right)^{*} a=a^{\boxplus} a^{2}=a$. That is $a R \subseteq a^{*} R$. Therefore the condition (2) is satisfied.

In [6, Theorem 16], for an operator $T \in L(X)$, where $X$ is a Banach space, Boasso proved that for a Moore-Penrose invertible operator $T, T$ is an EP operator if and only if there exists an invertible operator $P \in L(X)$ such that $T^{\dagger}=P T$. Inspired by this result, we get the following theorem.

Theorem 3.11. Let $a \in R^{\boxplus}$. Then the following are equivalent:

(1) $a \in R^{\mathrm{EP}}$;

(2) there exists a unit $u \in R$ such that $a^{\oplus}=u a$;

(3) there exists a left invertible element $v \in R$ such that $a^{\boxplus}=v a$;

(4) there exists an element $b \in R$ such that $a^{\boxplus}=b a$.

Proof. (1) $\Rightarrow(2)$ : Suppose $a \in R^{\mathrm{EP}}$, then $a \in R^{\oplus}$ and $a^{\boxplus}=a^{\#}$. Let $u=\left(a^{\#}\right)^{2}+1-a a^{\#}$. Since $u\left(a^{2}+1-a a^{\#}\right)=\left(a^{2}+1-a a^{\#}\right) u=1$, thus $u$ is a unit. And $u a=\left(\left(a^{\#}\right)^{2}+1-a a^{\#}\right) a=$ $a^{\#}=a^{\boxplus}$.

$(2) \Rightarrow(3)$ and $(3) \Rightarrow(4)$ are clear.

$(4) \Rightarrow(1)$ We know that $R a^{\oplus}=R a^{*}$ by the definition of the core inverse. From $a^{\oplus}=b a$ we get $R a^{\oplus} \subseteq R a$. Thus $R a^{*}=R a^{\oplus} \subseteq R a$. As a core invertible element is group invertible, we deduce that $a \in R^{\mathrm{EP}}$ by Theorem 3.7. 


\section{When a Moore-Penrose invertible element is an EP ele- ment}

Since any EP element is Moore-Penrose invertible, it is natural to ask when a MoorePenrose invertible element is an EP element. The concept of bi-EP was introduced by Hartwig and Spindelböck in [18] for complex matrices. They proved that for a complex $A \in \mathbb{C}_{n \times n}$, if $A$ is group invertible, then $A$ is an EP matrix if and only if $A$ is bi-EP. We give a generalization of the above result in Theorem 4.3. In this section, we give the definition of $n$-EP, which is a generalization of bi-EP. We show that any $n$-EP element is an EP element whenever this element is group invertible.

Definition 4.1. [18] An element $a \in R$ is called bi-EP if $a \in R^{\dagger}$ and $\left[a a^{\dagger}, a^{\dagger} a\right]=0$.

Definition 4.2. Let $n$ be a positive integer. An element $a \in R$ is called $n$-EP if $a \in R^{\dagger}$ and $\left[a^{n} a^{\dagger}, a^{\dagger} a^{n}\right]=0$.

Note that 1-EP is coincide with bi-EP.

In [14, Theorem 2.1], Mosić and Djordjević proved that $a \in R^{\mathrm{EP}}$ if and only if $a \in$ $R^{\#} \cap R^{\dagger}$ and $a^{n} a^{\dagger}=a^{\dagger} a^{n}$ for some $n \geqslant 1$. This result also can be found in [7, Theorem 2.4] by Chen. In the following theorem, we give a generalization of this result.

Theorem 4.3. Let $a \in R$ and $n$ be a positive integer. Then $a \in R^{\mathrm{EP}}$ if and only if $a \in R^{\dagger} \cap R^{\#}$ and $a$ is $n-E P$.

Proof. Suppose $a \in R^{\mathrm{EP}}$. Then $\left[a, a^{\dagger}\right]=0$, which gives $\left[a^{n} a^{\dagger}, a^{\dagger} a^{n}\right]=0$. That is $a$ is $n$-EP.

Conversely, suppose that $a \in R^{\dagger} \cap R^{\#}$ and $a$ is $n$-EP. Then we have

$$
a^{\dagger} a^{2 n} a^{\dagger}=a^{n}\left(a^{\dagger}\right)^{2} a^{n} .
$$

Pre-multiplication and post-multiplication of (4.1) by $a$ respectively now yields $a^{2 n} a^{\dagger}=$ $a^{n+1}\left(a^{\dagger}\right)^{2} a^{n}$, and $a^{\dagger} a^{2 n}=a^{n}\left(a^{\dagger}\right)^{2} a^{n+1}$. Thus

$$
\begin{aligned}
& a^{2 n-1} a^{\dagger}=a^{\#} a^{2 n} a^{\dagger}=a^{\#} a^{n+1}\left(a^{\dagger}\right)^{2} a^{n}=a^{n}\left(a^{\dagger}\right)^{2} a^{n} . \\
& a^{\dagger} a^{2 n-1}=a^{\dagger} a^{2 n} a^{\#}=a^{n}\left(a^{\dagger}\right)^{2} a^{n+1} a^{\#}=a^{n}\left(a^{\dagger}\right)^{2} a^{n} .
\end{aligned}
$$

By (4.2) and (4.3), we have $a^{2 n-1} a^{\dagger}=a^{\dagger} a^{2 n-1}$. Hence by [14, Theorem 2.1], we have $a \in R^{\mathrm{EP}}$.

Theorem 4.4. Let $a \in R^{\dagger}$. Then the following are equivalent:

(1) $a \in R^{\mathrm{EP}}$;

(2) there exists a unit $u \in R$ such that $a^{\dagger}=u a$;

(3) there exists a left invertible element $v \in R$ such that $a^{\dagger}=v a$.

Proof. (1) $\Rightarrow(2)$ : Suppose $a \in R^{\mathrm{EP}}$, then $a \in R^{\dagger}$ and $a^{\dagger}=a^{\#}$. Let $u=\left(a^{\#}\right)^{2}+1-a a^{\#}$. Since $u\left(a^{2}+1-a a^{\#}\right)=\left(a^{2}+1-a a^{\#}\right) u=1$, thus $u$ is a unit. And $u a=\left(\left(a^{\#}\right)^{2}+1-a a^{\#}\right) a=$ $a^{\#}=a^{\dagger}$.

$(2) \Rightarrow(3)$ is clear.

$(3) \Rightarrow(1)$ Suppose there exists a left invertible element $v \in R$ such that $a^{\dagger}=v a$. Then $1=t v$ for some $t \in R$ and $t a^{\dagger}=t v a=a$. Thus $R a^{\dagger} \subseteq R a$ and $R a \subseteq R a^{\dagger}$. Since $R a^{\dagger}=R a^{*}$ and $R a^{*}=R a^{\dagger}$, we deduce that $R a^{*} \subseteq R a$ and $R a \subseteq R a^{*}$. Therefore $R a=R a^{*}$, that is $a$ is an EP element. 
Remark 4.5. In Theorem 3.11 (4), we proved that for a core invertible element $a \in R$, $a \in R^{\mathrm{EP}}$ if and only if there exists an element $b \in R$ such that $a^{\boxplus}=b a$. The following example shows that this affirmation can not be obtained for a Moore-Penrose invertible element.

Recall that An infinite matrix $M$ is said to be bi-finite if it is both row-finite and column-finite.

Example 4.6. Let $R$ be the ring of all bi-finite matrices over in field $\mathbb{F}$ with transpose as involution and $e_{i, j}$ be the matrix in $R$ with 1 in the $(i, j)$ position and 0 elsewhere. Let $A=\sum_{i=1}^{\infty} e_{i+1, i}$ and $B=A^{*}$, then $A B=\sum_{i=2}^{\infty} e_{i, i}, B A=I$. So $A^{\dagger}=B$ and $A^{\dagger}=A^{\dagger} B A=$ $B^{2} A$. It is easy to check that $B^{2}$ is not left invertible and $A$ is not $\mathrm{EP}$ ( since $A B \neq B A$ ).

Proposition 4.7. Let $a \in R^{\dagger}$. Then the following are equivalent:

(1) $a \in R^{\mathrm{EP}}$;

(2) $\left[a^{\dagger} a, a\right]=\left[a^{\dagger}, a a^{\dagger}\right]=0$;

(3) $\left[a^{\dagger} a, a\right]=\left[a, a a^{\dagger}\right]=0$;

(4) $\left[a^{\dagger} a, a^{\dagger}\right]=\left[a^{\dagger}, a a^{\dagger}\right]=0$;

(5) $\left[a^{\dagger} a, a^{\dagger}\right]=\left[a, a a^{\dagger}\right]=0$.

Proof. (1) $\Rightarrow(2)-(3)$ : If $a \in R^{\mathrm{EP}}$, then $a a^{\dagger}=a^{\dagger} a$. Thus (2) and (3) are obvious.

$(2) \Rightarrow(1)$ Assume that (2) holds. Observe that $\left[a^{\dagger} a, a\right]$ implies that $a=a^{\dagger} a^{2} \in a^{*} R$ and $\left[a^{\dagger}, a a^{\dagger}\right]$ implies that $a^{\dagger}=a\left(a^{\dagger}\right)^{2} \in a R$, that is $a^{*} R \subseteq a R$ since $a^{\dagger} R=a^{*} R$. Thus, $a R=a^{*} R$, i.e., $a$ is EP.

$(3) \Rightarrow(1)$ : Assume that (2) holds. Observe that $\left[a^{\dagger} a, a\right]$ implies that $a=a^{\dagger} a^{2} \in a^{*} R$ and $\left[a, a a^{\dagger}\right]$ implies that $a=a^{2} a^{\dagger} \in R a^{\dagger}$, that is $R a \subseteq R a^{\dagger}$ since $R a^{\dagger}=R a^{*}$. We deduce that $R a \subseteq R a^{*}$, which is equivalent to $a^{*} R \subseteq a R$. Thus, $a R=a^{*} R$, i.e., $a$ is EP.

The equivalence between $(1) \Leftrightarrow(4) \Leftrightarrow(5)$ is similar to the proof of the equivalence between $(1) \Leftrightarrow(2) \Leftrightarrow(3)$.

Example 4.8. The condition $\left[a^{\dagger} a, a^{\dagger}\right]=0$ in Proposition 4.7 does not imply that $a$ is an EP element in general. Let $R, A$ and $B$ be same as Example 4.6 , then $A B=\sum_{i=2}^{\infty} e_{i, i}$, $B A=I$. So $A^{\dagger}=B$ and $\left[A^{\dagger} A, A^{\dagger}\right]=0$. But $A$ is not EP by $A B \neq B A$.

Lemma 4.9. [16] Let $a \in R$. Then the following are equivalent:

(1) $a \in R^{\mathrm{EP}}$;

(2) $a \in R^{\dagger}$ and $a R=a^{*} R$;

(3) $a \in R^{\dagger}$ and $R a=R a^{*}$.

Example 4.8 also shows that the equality $a R=a^{*} R$ in Lemma 4.9 cannot be replaced by inclusions $a R \subseteq a^{*} R$ or $a^{*} R \subseteq a R$.

Theorem 4.10. Let $a \in R^{\dagger}$. Then the following are equivalent:

(1) $a \in R^{\mathrm{EP}}$;

(2) $a R=a^{2} R$ and $\left[a^{\dagger} a, a^{\dagger}\right]=0$;

(3) $a R=a^{2} R$ and $\left[a^{\dagger} a, a\right]=0$;

(4) $a R=a^{2} R$ and $a R \subseteq a^{\dagger} R$;

(5) $a R=a^{2} R$ and $a R \subseteq a^{*} R$. 
Proof. (1) $\Rightarrow(2)-(5)$ : For a Moore-Penrose invertible element $a \in R, a \in R^{E P}$ if and only if $a a^{\dagger}=a^{\dagger} a$. Then (2)-(5) hold.

$(2) \Rightarrow(1)$ : Assume that (2) holds. Observe that $\left[a^{\dagger} a, a^{\dagger}\right]=0$ implies $a^{\dagger}=\left(a^{\dagger}\right)^{2} a$. Thus $R a^{*} \subseteq R a$ (Since $R a^{\dagger}=R a^{*}$ ). That is $a^{*}=r a$ for some $r \in R$. We deduce that $a^{*}=r a=r a a^{\dagger} a=a^{*} a^{\dagger} a$, applying involution on that last equality we obtain $a=a^{\dagger} a^{2}$. Therefore $a \in R^{\mathrm{EP}}$ by Theorem 3.7.

$(3) \Rightarrow(1)$ : It is clear that $\left[a^{\dagger} a, a\right]=0$ implies $a=a^{\dagger} a^{2}$. Thus $a \in R^{\mathrm{EP}}$ by Theorem 3.7 and the proof of $(2) \Rightarrow(1)$.

$(5) \Rightarrow(1):$ As $a R \subseteq a^{*} R$ is equivalent to $R a^{*} \subseteq R a$, we get $a \in R^{\mathrm{EP}}$ by the proof of $(2) \Rightarrow(1)$.

(4) $\Leftrightarrow(5)$ : It is clear by $a^{*} R=a^{\dagger} R$.

Similarly, we have the following theorem.

Theorem 4.11. Let $a \in R^{\dagger}$. Then the following are equivalent:

(1) $a \in R^{\mathrm{EP}}$;

(2) $R a=R a^{2}$ and $\left[a a^{\dagger}, a^{\dagger}\right]=0$;

(3) $R a=R a^{2}$ and $\left[a a^{\dagger}, a\right]=0$;

(4) $R a=R a^{2}$ and $R a \subseteq R a^{\dagger}$;

(5) $R a=R a^{2}$ and $R a \subseteq R a^{*}$.

\section{ACKNOWLEDGMENTS}

This research is supported by the National Natural Science Foundation of China (No. 11201063 and No. 11371089), the Specialized Research Fund for the Doctoral Program of Higher Education (No. 20120092110020); the Jiangsu Planned Projects for Postdoctoral Research Funds (No. 1501048B); the Natural Science Foundation of Jiangsu Province (No. BK20141327).

\section{References}

[1] A. Ben-Israel, T.N. Greville. Generalized Inverses: Theory and Applications. Wiley, Chichester, UK, 1977.

[2] O.M. Baksalary, G. Trenkler. Characterizations of EP, normal and Hermitian matrices. Linear Multilinear Algebra 56:299-304, 2006.

[3] O.M. Baksalary, G. Trenkler. Core inverse of matrices. Linear Multilinear Algebra 58(6):681-697, 2010.

[4] J. Benítez. Moore-Penrose inverses and commuting elements of $C^{*}$-algebras. J. Math. Anal. Appl. 345(2):766-770, 2008.

[5] K.P.S. Bhaskara Rao. The Theory of Generalized Inverses Over Commutative Rings. Taylor and Francis.

[6] E. Boasso. On the Moore-Penrose inverse, EP Banach space operators, and EP Banach algebra elements. J. Math. Anal. Appl. 339(2):1003-1014, 2008. 
[7] W.X. Chen. On EP elements, normal elements and partial isometries in rings with involution. Electron. J. Linear Algebra 23:553-561, 2012.

[8] R.E. Hartwig. Block generalized inverses. Arch. Retion. Mech. Anal. 61(3):197-251, 1976.

[9] R.E. Hartwig, J. Luh. A note on the group structure of unit regular ring elemments. Pacific J. Math. 71(2),449-461, 1977.

[10] R.E. Hartwig, K. Spindelböck. Matrices for which $A^{*}$ and $A^{\dagger}$ commmute. Linear Multilinear Algebra 14:241-256, 1984.

[11] J.J. Koliha, P. Patrício. Elements of rings with equal spectral idempotents. J. Aust. Math. Soc. 72(1):137-152, 2002.

[12] D. Mosić, D.S. Djordjević, J.J.Koliha. EP elements in rings. Linear Algebra Appl. 431:527-535, 2009.

[13] D. Mosić, D.S. Djordjević. Partial isometries and EP elements in rings with involution. Electron. J. Linear Algebra 18:761-772, 2009.

[14] D. Mosić, D.S. Djordjević. New characterizations of EP, generalized normal and generalized Hermitian elements in rings. Appl. Math. Comput. 218:6702-6710, 2012.

[15] X. Mary. On generalized inverses and Green's relations. Linear Algebra Appl. 434:1836-1844, 2011.

[16] P. Patrićio, R. Puystjens. Drazin-Moore-Penrose invertiblity in rings. Linear Algebra Appl. 389:159-173, 2004.

[17] D.S. Rakić, Nebojša Č. Dinčić, D.S. Djordjević. Group, Moore-Penrose, core and dual core inverse in rings with involution. Linear Algebra Appl. 463:115-133, 2014.

[18] H. Schwerdtfeger. Introduction to Linear Algebra and the Theory of Matrices. P. Noordhoff Groningen, 1950.

[19] J. von Neumann. On regular rings. Proc. Nati. Acad. Sci. U.S.A. 22(12):707-713, 1936. 